\title{
Impulse Amplitude Discrimination Circuit and Its Application to Spontaneous Discharge Analysis
}

\author{
Itsuo Yamaura* and Shiko Chichibu ${ }^{\dagger}$ \\ Microwave Technology Laboratory (Prof. Y. Mushiake), \\ Department of Electrical Communications, Faculty of Engineering, and \\ Department of Physiology (Prof. K. Tasaki), School of Medicine. \\ Tohoku University, Senciai
}

\begin{abstract}
A simple electronic device to discriminate amplitudes of action potentials was described. The principle of the impulse amplitude discrimination circuit (IADC) was to use the time lag in the falling phase of the action potential and the application of NOT-AND gate in the circuit. An application of IADC to the analysis of spontaneous discharges from the crayfish abdominal ganglion, and the detection of microwave effect was presented.
\end{abstract}

Frequency and interval measurements are very common methods used for analysis of information transmissions in the excitable tissues and are applied in various fields $s^{1,2}$ of electrophysiology and of clinical medicine. In order to complete the frequency and interval analysis in a short time, it is desirable to use special devices, such as electronic computers and frequency analyzers, and so the application of these instruments is currently done in many laboratories. There are several reviews on these problems and techniques. ${ }^{3,5}$

For the need of pulse-height analysis in various fields of sciences, a number of analyzers have been developed especially in the nuclear science and in the electronics in association with the industrial technology. 2,3,5,7 Almost all of these pulse-height analyzers were designed specially for physical phenomena occurring within nanoseconds or so, ${ }^{2,4.7}$ and are not convenient for a use in the bioelectrical works.

There are many difficult problems in the frequency analysis of action potentials. Among them, the most important need is to record a series of isolated unitary discharges without contamination of smaller impulses. This is often difficult because of the limits in the characteristics of instruments and of the complex orientation of cells.

A type of pulse-height analyzer specially designed for the phenomena occurring in milliseconds' range was developed in order to avoid these difficulties, and to

Received for publication, June 24, 1967.

* Present address: The Research Institute of Applied Flectricity, Hokkaido University, Sapporo.

$\dagger$ Present address: Department of Physiology, Tohoku University School of Dentistry, Sendai. 
facilitate an analysis of spike (impulse) intervals in a complicated series with impulses of various amplitudes.

In the present paper, the principle of this circuit and its application to the analysis of microwave effect on the spontaneous discharges in the crayfish ganglion were described.

\section{Methods}

The impulse amplitude discrimination circuit (IADC) was constructed mainly with transistors and diodes, and was operated with dry cells. The block diagram of IADC was shown in Fig. $1 \mathrm{~B}$, and the arrangement of the equipment for data processing were illustrated in Fig. $1 \mathrm{~A}$.

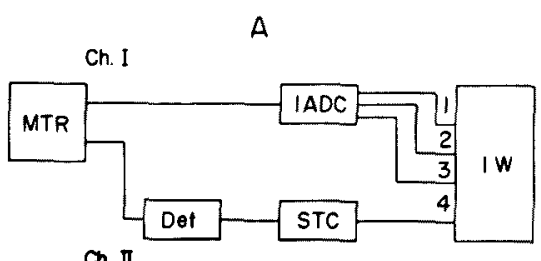

ch. II

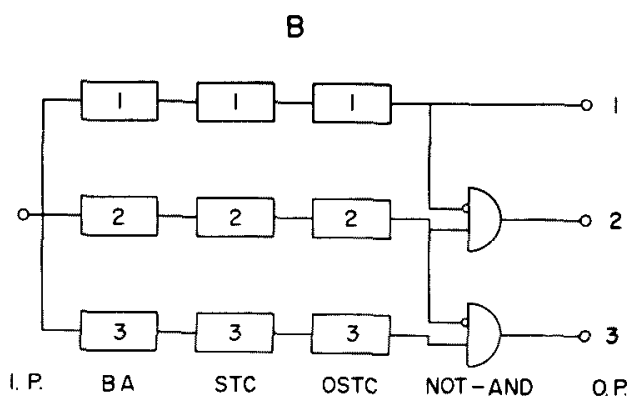

Fig. 1. Block diagram of the data processing (A), and of the impulse amplitude discrimination circuit (B). In A, MTR; magnetic tape recorder. Ch. I and Ch. II are channels in MTR. IADC; the impulse amplitude discrimination circuit. Det; detector circuit. STC; Schmitt trigger cireuit. IW; ink-writer. $1,2,3$ and 4 are lines in IW. In $B$, I.P.; input from MTP. BA; buffer amplifiers. STC; Schmitt trigger circuits, NOT-AND; not-and gate. OSTC; one shot trigger circuits. O.P.; output to each galvanometers in IW.

In this IADC, potential differences at the falling phase of action potentials were used instead of those of the rising phase of action potentials with delay and comparing eircuits. In Fig. 2, the input-output relationships in each stages of IADC were schematically illustrated.

At 1 and 2 in Fig. $2 \mathrm{~A}$ slicing levels of action potentials by adjusting thresholds in the circuit were shown. The base-line or zero level was indicated by 3 in this figure, so that the largest action potential was sliced at three different levels (including zero). 


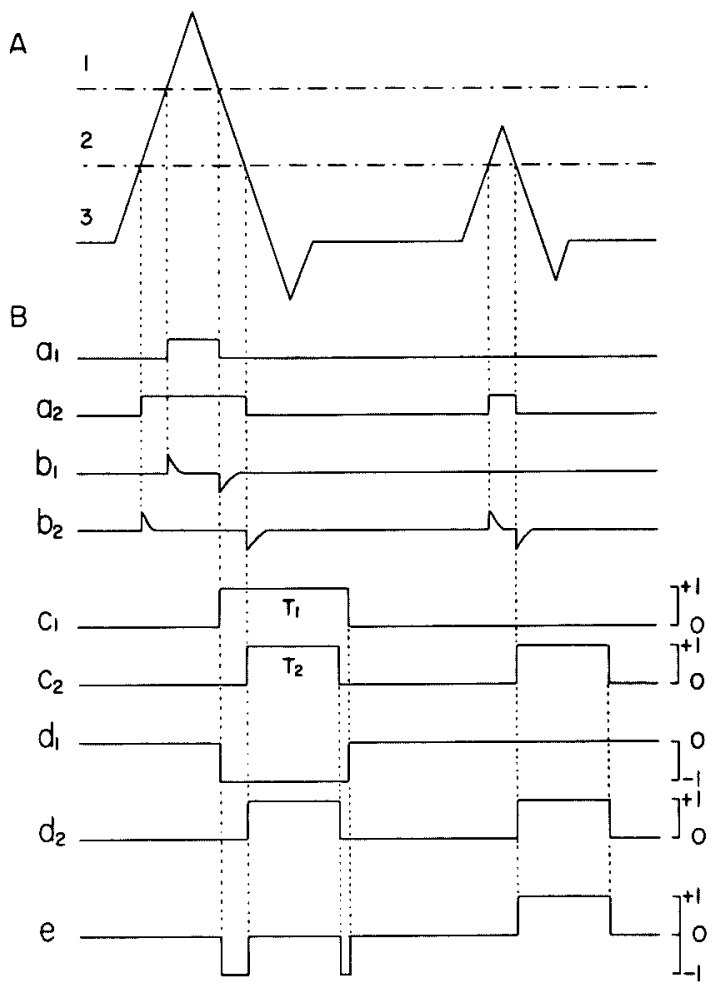

Fig. 2. A: Schematic presentation of two action potentials with one large and one small amplitude. 1, 2 and 3 indicate amplitude slicing thresholds in IADC. B: Schematized input-output relationship in IADC. $a_{1}$ and $a_{2}$; output from STC. 1 and 2 refer to STC 1 and 2. $b_{1}$ and $b_{2}$; input pulse to OSTC. $c_{1}$ and $c_{2}$; output frcm OSTC. $T_{1} T_{2} . c_{1}$ goes to line 1 of IW. $d_{1}$ and $d_{2}$; input to NOT-AND. $d_{1}$ was obtained by reversal of the phase in $c_{1} \cdot d_{2}$ is the same to $c_{2}$. e; output from O.P. 2 , and goes to line 2 of IW.

Signals (impulses) coming out from the output of the magnetic tape recorder channel I were fed simultaneously into three parallel lines of IADC at first. The possible cross-talks and interactions between the lines were eliminated by placing a group of buffer amplifiers in the first stage. Thresholds for different levels of impulse amplitude were chosen and set separately in each line of IADC.

With this pre-setting, the sliced impulses at different levels then drive the Schmitt trigger circuits (STC) to generate square pulses $\left(a_{1}\right.$ and $\left.a_{2}\right)$. These pulses have comparable durations with those of impulses sliced at the pre-set levels. These pulses are then differentiated at the C- $R$ networks with short time constant, and become the transient spikes as shown in $b_{1}$ and $b_{2}$.

These spikes are then transmitted to the input of the next one-shot trigger circuit in each line. Of these spikes, negative spikes only are able to fire square pulses with previously-determined durations at the one-shot trigger (OSTC). Predetermined durations of pulses, $\mathrm{T}_{1}$ and $\mathrm{T}_{2}$, must satisfy the following points; 
$T_{1}$ and $T_{2}$ have enough durations to give sufficient time for driving of galvanometers in the ink-writer. In the following experiment in this paper, $\mathrm{T}_{1}$ and $\mathrm{T}_{2}$ were set as $3.8 \mathrm{msec}$ and $3.5 \mathrm{~m} \mathrm{sec}$. These values were chosen from the following reasons: The duration of the action potential with extracellular recording was close to 0.5 msec. When sliced into three different levels, the falling phase within one level was less than $0.3 \mathrm{msec}$. The pulse duration of $3 \mathrm{msec}$ was the minimum for galvanometer driving. The mean value of the discharge frequency of the ganglion was not high, and usually less than $40 \mathrm{impulses} / \mathrm{sec}$. If the number of shorter intervals considered to be less than $4 \mathrm{msec}$ is small, it is preferable to use the value around 3.5 msec for $T_{1}$ and $T_{2} . \quad c_{1}$ and $c_{2}$ are the output from OSTC, and $c_{1}$ is displayed in one line of the ink-writer. This line gives the record of the spikes/impulses having the largest amplitude.
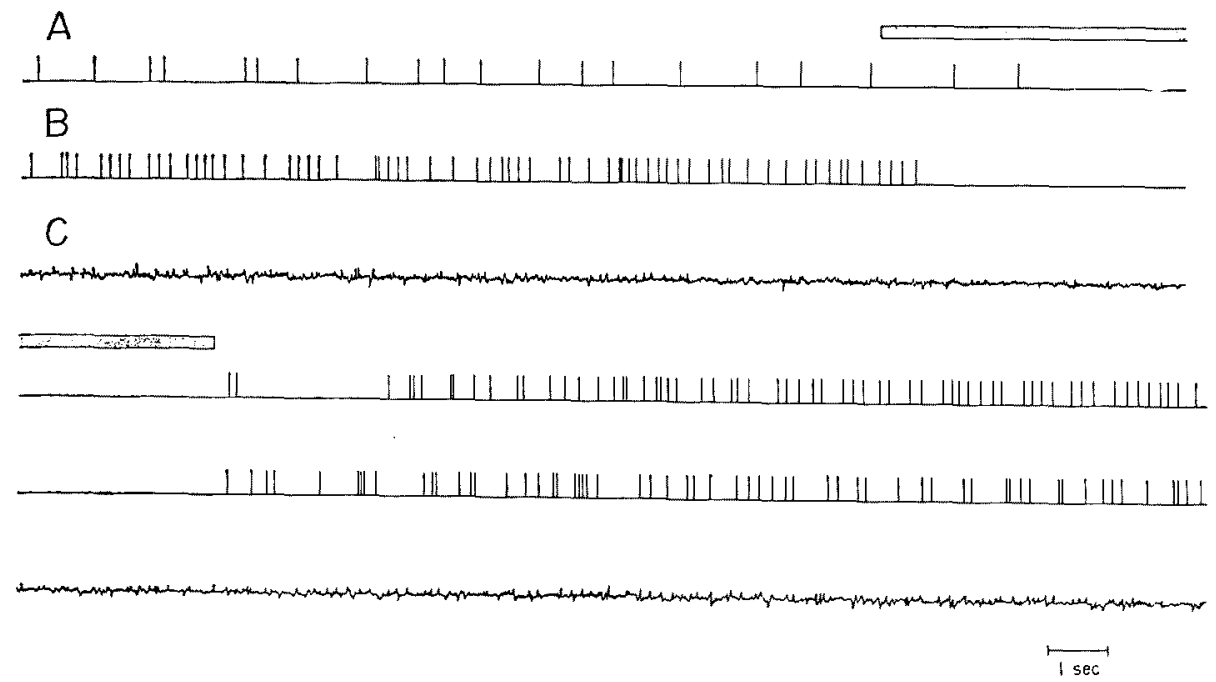

Fig. 3. An example of IADC used in erayfish ganglionic discharge analysis. A: The highest level action potentials (over $200 \mu \mathrm{V}$ ). B: Middle level spikes (100 to $200 \mu \mathrm{V}$ amplitude). C: Original record displayed with the ink-writer. Dotted horizontal bar is the duration of microwave irradiation, $100 \mu \mathrm{W} / \mathrm{mm}^{3}$.

At the same time, the phase of $c_{1}$ is reversed and put to the input of a NOTAND gate with $c_{2}$. e is the output of this NOT-AND gate, and is led to another line of the ink-writer. Connection is made to display positive pulses only.

With this performance, spikes (impulses) having different amplitudes are displayed separately on the record as shown in Fig. 3.

Though it is convenient for the experiment, there is still several limitations to be improved in future. One of them is that this circuit is not suitable for analysis of high frequency discharges. This disadvantage are mostly due to the durations, $\mathrm{T}_{1}$ and $\mathrm{T}_{2}$. When $\mathrm{T}_{1}$ is set at $3.8 \mathrm{msec}$, the maximum frequency applicable to this circuit is 263.2 impulses per sec, and the practical working range of the frequency 
characteristic will be up to 130 impulses per sec. This figure of the frequency characteristic is quite satisfactory as far as the recording is displayed with the inkwriter, because the frequency characteristic of ordinary ink-writers are about 100 to 120 cycles with $-3 \mathrm{db}$.

The ganglionic discharges of crayfish were studied with this IADC and the effect of microwave irradiation was detected in the series of impulses having different amplitudes. The details of the experimental techniques except for the method of data processing were the same as those described in the preceding paper. ${ }^{8}$ The superhigh frequency electric field of $11 \mathrm{Gc}$ and $100 \mu \mathrm{W} / \mathrm{mm}^{3}$ available power was used in the experiments.

Experiments were carried out at temperatures of $15^{\circ}$ to $20^{\circ} \mathrm{C}$.

\section{Results}

It is known that the microwave irradiation produced several kinds of responses in biological tissues, but the cellular mechanism of the microwave effect is not known sufficiently. ${ }^{1}$

As described in our preceding paper, ${ }^{8}$ the microwave irradiation caused a biphasic change of impulse frequencies in the crustacean ganglion. When the record was taken directly with an ordinary ink-writer without IADC, it was difficult to find out the irradiation effect in every experiment. The trouble was caused by the contamination of smaller impulses and by the insufficiency of the power to drive the galvanometer. An example was illustrated in Fig. $3 \mathrm{C}$. When the same record was analyzed with IADC, the impulses in the high level and in the

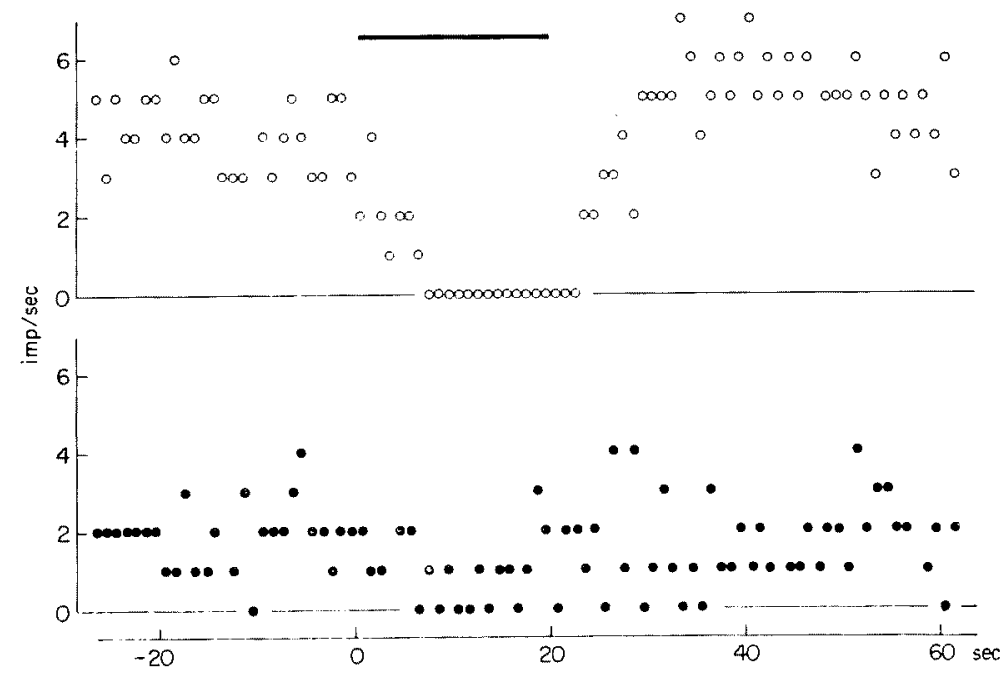

Fig. 4. Crayfish ganglionic discharge frequencies and the microwave irradiation (100 $\mathrm{W} /$ $\left.\mathrm{mm}^{3}\right)$. Top: action potentials with amplitudes over $400 \mu \mathrm{V}$. Bottom: middle range action potentials $(200-300 \mu \mathrm{V})$. Black bar: duration of the irradiation. 


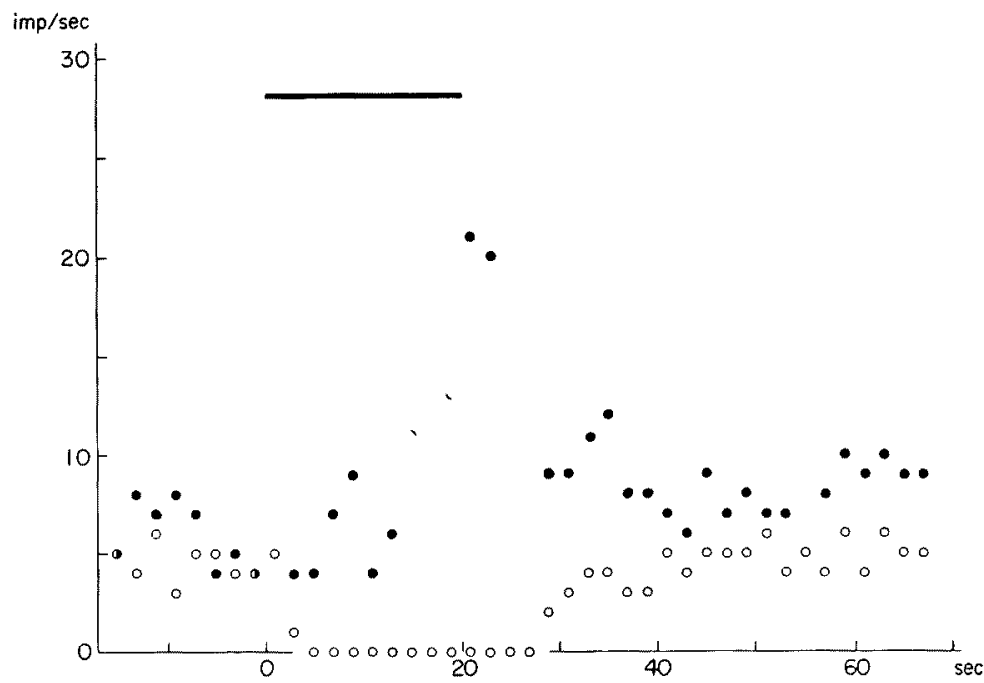

Fig. 5. Crayfish ganglion. Open circles refer to large impulses (over $400 \mu \mathrm{V}$ ). Filled circles are from the middle level $(150-400 \mu \mathrm{V})$. Black bar: duration of the irradiation.

middle level are separately demonstrated as shown in Fig. $3 \mathrm{~A}$ and $\mathrm{B}$.

Figs. 4 and 5 were constructed in the same way by counting the number of impulses per sec in each level. In Fig. 4, open circles in the top show the numbers of large impulses with amplitude of one kind in the cathode ray oscillographic monitoring. It is very possible that the frequency changes are the result of the microwave irradiation on one nerve cell. In the bottom of the same figure, the filled circles were obtained from the middle level, and the impulses at this level contained several kinds of impulses with different amplitudes within the level. In this middle level, the effect was not shown so clear as in the high level. This difference may come from the desynchronization of activities in different cells.

Another example with IADC is illustrated in Fig. 5. The response in the high level (open circles) shows a clear decrease in impulse frequency, but the one in the middle level (filled circles) is not distinct.

\section{Comments}

The use of the IADC was very effective in the studies on the effect of microwave irradiation on the crayfish ganglion. The IADC was designed for the analysis of nerve impulses, and was worked well for selection of impulses having amplitudes of a narrow range. It is not difficult to add another line of IADC, and to increase the number of discriminated levels according to the experimental requirement.

\section{Acknowledgment}

We are indebted to Drs. Y. Mushiake, K. Tasaki and S. Adachi for their encouragements, supports and suggestions. 


\section{References}

1) Boysen, J.E. A review of unanswered biological hazard operational problems. In: Biological effects of microuave radiation. I, edited by Mary F. Peyton, Plenum Press, N.Y., 1961, p. 309.

2) Higinbotham, W.A. Today's pulse-height analyzers. Nucleonics, 1956, 14, 61-64.

3) Katz, G., Webb. G. \& Sorem, A. Recording and display. In: Physical techniques in biological research. V, edited by W.L. Nastuk, Academic Press, New York and London, 1964, p. 373.

4) Lamonds, H.A. Fast differential analyzer. Nucleonics, 1956, 14, 86-90.

5) MeCann, G.D. \& Ray, C.B. Computers and data processing for nervous system research. IEEE Transac. on Bio-Medical Electronics, 1963, BME-10, 48-56.

6) Sumita, K., Kato, K. \& Furukawa, T. Atomic energy measurement. Series on the industrial electronic apparatus (Jap.), Nikkan-Kogyoshinbun Co., Tokyo, 1966, p. 67.

7) Van Rennes, A.B. Single-channel differential analyzers. Pulse-amplitude analysis in nuclear research. Part II, Nucleonics, 1952, 10, 22-28.

8) Yamaura, I. \& Chichibu, S. Super-high frequency electric field and crustacean ganglionic discharges. Tohoku J. exp. Med., 1967, 93, 249-259. 